\title{
Asenkron Motorların GKF Tabanlı Model Öngörülü Moment Kontrolü
}

\author{
Yunus Emre Altınışık ${ }^{1}$, Rıdvan Demir ${ }^{2 *}$ \\ ${ }^{1}$ Niğde Ömer Halisdemir Üniversitesi, Mühendislik Fakültesi, Elektrik Elektronik Mühendisliği Bölümü, Niğde, Türkiye, (ORCID: 0000-0002-9666-7533), \\ yunusemre431@hotmail.com \\ 2* Kayseri Üniversitesi, Mühendislik Mimarlık ve Tasarım Fakültesi, Elektrik Elektronik Mühendisliği Bölümü, Kayseri, Türkiye (ORCID: 0000-0001-6509-9169), \\ ridvandemir@kayseri.edu.tr
}

(Uluslararası Araştırma-Geliştirme ve Tasarım Konferansı - 15-18 Aralık 2021)

(DOI: 10.31590/ejosat.1041552)

ATIF/REFERENCE: Altınışı, YE., \& Demir, R. (2021). Asenkron Motorların GKF Tabanlı Model Öngörülü Moment Kontrolü. European Journal of Science and Technology, (32), 858-863.

$\ddot{O} \mathbf{z}$

$\mathrm{Bu}$ çalışmada rotor direncinin kestirimi ile kontrol başarımı iyileştirilmiş hız-algılayıcısız model öngörülü moment kontrol (model predictive torque control, MPTC) tabanlı asenkron motor (ASM) sürücüsü sunulmuştur. Bu amaçla, MPTC'nin yüksek başarımlı hız kontrolü için gerekli olan hız ve akı bilgisine ek olarak rotor direnci kestirimi; girişinde ölçülen stator gerilim ve akımlarını kullanan genişletilmiş Kalman filtresi (GKF) tarafından gerçekleştirilmiştir. GKF tarafından kestirilen rotor direnci MPTC sistemi içerisine her bir örnekleme adımında güncellenerek parametre değişimlerinden kaynaklanan bozulmalar azaltılmıştır. Tasarlanan GKF algoritması ve bu algoritmayı kullanan MPTC tabanlı ASM sürücüsü yük momenti ve rotor direnci değişimlerini içeren zorlayıcı senaryolar altında geniş bir hız aralığında benzetim ortamında test edilmiş ve doğrulanmıştır. Elde edilen benzetim sonuçları, GKF algoritmasının yüksek kestirim başarımına, buna bağlı olarak hız-algılayıcısız MPTC temelli ASM sürücüsünün ise yüksek kontrol başarımına sahip olduğunu onaylamaktadır.

Anahtar Kelimeler: Model öngörülü kontrol, Asenkron motorlar, Genişletilmiş Kalman filtresi.

\section{EKF Based Model Predictive Torque Control of Induction Motors}

\begin{abstract}
In this study, speed-sensorless model predictive torque control (MPTC) based induction motor (IM) drive that control performance is improved by estimating rotor resistance is presented. For this purpose, in addition to the speed and flux information required for highperformance speed control of MPTC, estimation of rotor resistance was realized by Extended Kalman filter (EKF) that uses the stator currents and voltages which are measured as inputs. The rotor resistance estimated by GKF is updated to the MPTC system at each sampling step, reducing the deteriorates caused by parameter changes. Designed EKF algorithm and MPTC based IM driver which used this algorithm is tested and confirmed in simulation environment over a wide speed range under challenging scenarios including load torque and rotor resistance variations. The obtained simulation results confirm that the GKF algorithm has high estimation performance, and accordingly the speed-sensorless MPTC-based ASM drive has high control performance.
\end{abstract}

Keywords: Model predictive control, Induction motors, Extended Kalman filter.

\footnotetext{
*Sorumlu Yazar: ridvandemir@kayseri.edu.tr
} 


\section{Giriş}

Sağlam yapıları, az bakım gereksinimleri, düşük maliyetleri, zorlu ortamlarda çalışabilme ve güvenilirlik gibi özelliklerinden dolayı asenkron motorlar (ASM'ler) değişken hız/konum ve/veya moment kontrolünü gerektiren endüstriyel ve elektrikli araç uygulamalarında yaygın olarak tercih edilmektedirler. ASM'lerin yüksek başarımlı hız/konum ve/veya moment kontrolü için alan yönlendirmeli kontrol (AYK) ya da vektör kontrol (VK), doğrudan moment kontrol (DMK) ve model öngörülü kontrol (model predictive control, MPC) gibi yüksek başarımlı kontrol yöntemleri literatüre önerilmiştir (Altınışık vd., 2021).

MPC yöntemi AYK ve DMK'ya kıyasla sunduğu üstünlükler nedeniyle elektrik motor sürücü sistemleri ve güç elektroniği konularında çalışan araştırmacılar tarafından ilgi görmektedir (Türker vd., 2018). Model öngörülü moment kontrolü (model predictive torque control-MPTC) MPC'lerin bir türüdür. MPTC'nin ana çıkış noktası evirici ve motorun belirgin modelinin kullanılması ile öngörülen elektromanyetik momenti ve stator akısını içeren maliyet fonksiyonunu eniyileyen gerilim vektörünün seçilmesine dayanmaktadır. MPTC uygulanmasının basit olması, kolay anlaşılması, kısıtlamaların dahil edilebilmesi ve çok değişkenli doğrusal olmayan sistemleri ele alabilmesi gibi üstünlüklere sahiptir. Bununla birlikte, fazla işlem yükü bu yöntemin olumsuz yönü olarak değerlendirilebilir. Ancak, günümüzde gelişen mikro işlemci mimarisi ile bu olumsuzluğun üstesinden gelinmiştir.

ASM'lerin hız-algılayıcısız kontrolü, doğrusal olmayan modeli, bilinmeyen yük girişleri ve çalışma koşulları ile değerleri değişen parametreleri nedeniyle karmaşık hale gelmektedir. Hızalgilayıcısız MPTC ASM'nin rotor hizı ile birlikte stator/rotor akılarının kestirilmesine ihtiyaç duyulmaktadır. Ancak frekans ve sıcaklık gibi ASM'nin çalışma koşulları ile rotor ve stator dirençlerinde meydana gelen bu değişimler, akı ve hız kestirimlerde bozulmalara neden olmaktadır.

ASM'lerin yüksek başarımlı hı-algılayıcısız kontrolünü gerçekleştirmek için modele uyarlamalı sistemler (Wang vd., 2014; Xie vd., 2021), kayma kipli (sliding mode) gözlemleyicisi (Lu \& Zhao, 2018; Zhao vd., 2019), tam dereceli gözlemleyici (Nguyen vd., 2021; Yin vd., 2019), genişletilmiş Luenberger gözlemleyicisi (Yan \& Song, 2020) ve genişletilmiş Kalman filtresi (GKF) (Demir \& Barut, 2018; Zerdali \& Demir, 2021) gibi model tabanlı kestiriciler ya da gözlemleyiciler tanıtılmıştır. Literatüre önerilen kestirim yöntemlerinden GKF'ler ölçme gürültülerini göz önünde bulundurarak parametre ve durum kestirim problemini olasıl bir yaklaşım ile gerçekleştirmektedir.

Hız-algılayıcısız sürücüler için parametre ve durum kestirimini gerçekleştiren GKF gözlemleyicilerin kullanıldığı çalışmalara bakıldığında; kontrol sistemleri için gerekli olan hız ve akı kestrimlerine ek olarak stator direnci ve rotor direnci kestirimi (Altınışık vd., 2021)'de önerilen anahtarlamalı GKF gözlemleyicisi ile, (Demir \& Barut, 2018)'de ise GKF ve MUS'un paralel çalıştırılmasıyla kestirilmiştir.

MPTC yönteminin zamanla değişen parametrelerden kaynaklı kontrol başarımında meydana gelen bozulmaları önlemek için gerçekleştirilen çalışmalar literatürde mevcuttur (Yan vd., 2019; Zerdali vd., 2020). Zerdali vd. (2020)'de yük girişi ve stator direnci değişimlerinden kaynaklanan bozulmalar uyarlamalı GKF gözlemleyicisi tarafindan kestirilen yük momentinin hız çevrimi içerisine, stator direncinin ise MPTC sistemine güncellenmesi ile iyileştirilmiştir.

$\mathrm{Bu}$ çalışmanın temel katkısı rotor direnci değişimlerine karşı başarımı iyileştirilmiş hız-algılayıcısız MPTC tabanlı sürücü sistemini literatüre tanıtmaktır. Bu amaçla hız-algılayıcısız MPTC sistemi için gerekli olan akı ve hız bilgisine ek olarak rotor direncinin kestirimi eş-zamanlı olarak GKF gözlemleyicisi ile gerçekleştirilmiştir. GKF gözlemleyicisinde kestirilen rotor hızı ve rotor direnci sabit parametre olarak göz önünde bulundurulmuştur. Kestirilen rotor direnci MPTC tabanlı sürücü sistemi içerisine her bir örnekleme adımında güncellenmiş ve böylece sürücü sisteminin başarımı çalışma koşulları nedeniyle rotor direncinde meydana gelebilecek değişimlere karşı iyileştirilmiştir. MPTC tabanlı sürücü sistemi rotor direnci ve yük momentinde meydana getirilen değişiklikler ile üretilen zorlayıcı senaryolar altında geniş bir hız aralığı için benzetim çalışmaları ile test edilmiş ve doğrulanmıştır. Benzetim çalışmalarından elde edilen sonuçlar, MPTC tabanlı sürücü sisteminin yüksek kontrol başarımına, GKF gözlemleyicisinin ise yüksek kestirim başarımına sahip olduğunu göstermektedir.

$\mathrm{Bu}$ çalışma altı bölümden oluşmuştur. Bölüm 2'de iki seviyeli-gerilim kaynaklı evirici (two level-voltage source inverter, (2L-VSI)) tarafindan beslenen ASM'nin matematiksel modeli verilmiştir. GKF gözlemleyicisi ve Hı-algılayıcısız MPTC tabanlı ASM sürücüsü sırasıyla Bölüm 3 ve Bölüm 4'te sunulmuştur. Benzetim çalışmaları Bölüm 5 'te gösterilmiş ve son olarak sonuç Bölüm 6'da verilmiştir.

\section{2L-VSI tarafindan beslenen ASM'nin matematiksel modeli}

ASM'lerin stator duran eksenindeki matematiksel modeli aşağıdaki genel formda verilebilir.

$$
\begin{gathered}
\dot{\mathbf{x}}_{t}=\mathbf{f}\left(\mathbf{x}_{t}, \mathbf{u}_{t}\right)+\mathbf{w}_{t} \\
\mathbf{z}_{t}=\mathbf{h}\left(\mathbf{x}_{t}\right)+\mathbf{v}_{t}
\end{gathered}
$$

Burada $\mathbf{f}$, girişler ve durumların doğrusal olmayan fonksiyonu; $\mathbf{u}_{t}$ kontrol giriş vektörü; $\mathbf{x}_{t}$ durum vektörü; $\mathbf{w}_{t}$ sistem gürültüsü; $\mathbf{h}$ çıkışların fonksiyonu; $\mathbf{v}_{t}$ ise ölçüm gürültüsü olarak tanımlanabilir.

ASM'in Denklem (1)'de verilen matematiksel modelindeki vektörler aşağıda gösterilmiştir.

$$
\begin{aligned}
& \mathbf{x}_{k}=\left[\begin{array}{lllll}
i_{s \alpha} & i_{s \beta} & \psi_{r \alpha} & \psi_{r \beta} & \omega_{m}
\end{array}\right]^{T} \\
& \mathbf{u}_{t}=\left[\begin{array}{ll}
v_{s \alpha} & v_{s \beta}
\end{array}\right]^{T}, \quad \mathbf{h}=\left[\begin{array}{ll}
i_{s \alpha} & i_{s \beta}
\end{array}\right]^{T} \\
& \mathbf{f}=\left[\begin{array}{c}
-\left(\frac{R_{s}}{L_{\sigma}}+\frac{R_{r} L_{m}^{2}}{L_{\sigma} L_{r}^{2}}\right) i_{s \alpha}+\frac{R_{r} L_{m}}{L_{\sigma} L_{r}^{2}} \psi_{r \alpha}+\frac{L_{m} p_{p}}{L_{\sigma} L_{r}} \omega_{m} \psi_{r \beta}+\frac{v_{s \alpha}}{L_{\sigma}} \\
-\left(\frac{R_{s}}{L_{\sigma}}+\frac{R_{r} L_{m}^{2}}{L_{\sigma} L_{r}^{2}}\right) i_{s \beta}+\frac{R_{r} L_{m}}{L_{\sigma} L_{r}^{2}} \psi_{r \beta}-\frac{L_{m} p_{p}}{L_{\sigma} L_{r}} \omega_{m} \psi_{r \alpha}+\frac{v_{s \beta}}{L_{\sigma}} \\
\frac{R_{r} L_{m}}{L_{r}} i_{s \alpha}-\frac{R_{r}}{L_{r}} \psi_{r \alpha}-p_{p} \omega_{m} \psi_{r \beta} \\
\frac{R_{r} L_{m}}{L_{r}} i_{s \beta}-\frac{R_{r}}{L_{r}} \psi_{r \beta}+p_{p} \omega_{m} \psi_{r \alpha} \\
\frac{3 p_{p} L_{m}}{2 J_{t} L_{r}}\left(\psi_{r \alpha} i_{s \beta}-\psi_{r \beta} i_{s \alpha}\right)-\frac{B_{t}}{J_{t}} \omega_{m}-\frac{\tau_{l}}{J_{t}}
\end{array}\right]
\end{aligned}
$$


Burada $v_{s \alpha}, v_{s \beta}, i_{s \alpha}$ ve $i_{s \beta}$ stator gerilim ve akımlarının stator duran eksen takımındaki bileşenlerini; $\psi_{r \alpha}$ ve $\psi_{r \beta}$ rotor akılarının stator duran eksen takımındaki bileşenlerini; $p_{p}$ kutup çifti sayısını; $\omega_{m}$ rotor mekanik açısal hızını; $L_{s}, L_{r}$ ve $L_{m}$ stator, rotor ve mıknatıslama endüktanslarını; $L_{\sigma}=\sigma L_{s}=L_{s}-L_{m}^{2} / L_{r}$ stator geçici endüktansın1; $R_{S}$ ve $R_{r}$ stator ve rotor dirençlerini; $\sigma$ kaçak faktörünü; $J_{t}$ ve $B_{t}$ sırasıyla ASM ve yükün toplam eylemsizliği ile toplam viskoz sürtünme terimini; $\tau_{l}$ ise yük momentini ifade etmektedir.

Yukarıda genel modeli verilen ASM, Şekil 1a'da gösterilen 2L-VSI tarafindan beslenir ve 2L-VSI'nın anahtarlama durumu $\vec{S}$ ise aşağıdaki gibi ifade edilebilir.

$$
\vec{S}=\frac{2}{3}\left(S_{a}+\vec{a} S_{b}+\vec{a}^{2} S_{c}\right)
$$

burada $\vec{a} \triangleq e^{j 2 \pi / 3}$ ve $S_{x} \in\left\{S_{a}, S_{b}, S_{c}\right\}$ her bir koldaki anahtarların AÇIK/KAPALI durumlarını gösterir. İnverter çıkış gerilimi $\vec{v}_{s}$, anahtarlama durumu $\vec{S}$ kullanılarak aşağıdaki gibi ifade edilebilir:

$$
\vec{v}_{s}=V_{d c} \vec{S}
$$

burada $V_{d c}$, dc-bağlantı gerilimidir. 2L-VSI dikkate alındığında, denklem (3) sekiz anahtarlama durumu için sonlu kontrol kümeleri adı verilen yedi farklı gerilim vektörü verir. Bu gerilim vektörleri Şekil 1b'de görülebilir.

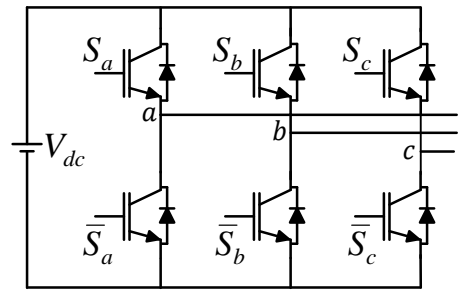

(a)

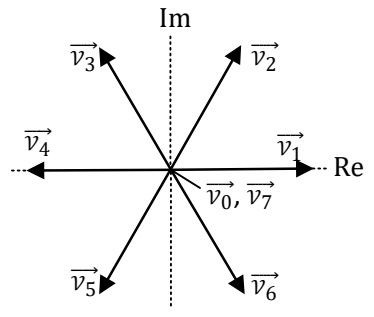

(b)
Şekil 1. (a) İki seviyeli gerilim kaynakl evirici, (b) gerilim vektörleri

\section{GKF Gözlemleyicisi}

$i_{s \alpha}, i_{s \beta}, \psi_{r \alpha}, \psi_{r \beta}, \omega_{m}$, ve $R_{r}$ 'nin eş-zamanlı kestirimini gerçekleştiren GKF gözlemleyicisinin kullandığı ASM modeli; denklem (1)'de genel formu verilen ASM modelinin $\omega_{m}$ ve $R_{r}$ ile genişletilmesi ile meydana getirilmiştir. GKF gözlemleyicisinde kullanılacak genişletilmiş modelde $\omega_{m}$ ve $R_{r}$ kestirilecek sabit parametre olarak dikkate alınmaktadır. Gözlemleyicinin kullandığı ASM modelindeki vektörler aşağıda sunulmuştur.

$$
\begin{aligned}
& \mathbf{x}_{o, t}=\left[\begin{array}{lllllll}
i_{s \alpha} & i_{s \beta} & \psi_{r \alpha} & \psi_{r \beta} & \omega_{m} & R_{r}
\end{array}\right]^{T}, \\
& \mathbf{u}_{o, k}=\left[\begin{array}{ll}
v_{s \alpha} & v_{s \beta}
\end{array}\right]^{T}, \mathbf{h}_{o}=\left[\begin{array}{ll}
i_{s \alpha} & i_{s \beta}
\end{array}\right]^{T}, \\
& \mathbf{f}_{o}=\left[\begin{array}{c}
-\left(\frac{R_{s}}{L_{\sigma}}+\frac{R_{r} L_{m}^{2}}{L_{\sigma} L_{r}^{2}}\right) i_{s \alpha}+\frac{R_{r} L_{m}}{L_{\sigma} L_{r}^{2}} \psi_{r \alpha}+\frac{L_{m} p_{p}}{L_{\sigma} L_{r}} \omega_{m} \psi_{r \beta}+\frac{v_{s \alpha}}{L_{\sigma}} \\
-\left(\frac{R_{s}}{L_{\sigma}}+\frac{R_{r} L_{m}^{2}}{L_{\sigma} L_{r}^{2}}\right) i_{s \beta}+\frac{R_{r} L_{m}}{L_{\sigma} L_{r}^{2}} \psi_{r \beta}-\frac{L_{m} p_{p}}{L_{\sigma} L_{r}} \omega_{m} \psi_{r \alpha}+\frac{v_{s \beta}}{L_{\sigma}} \\
\frac{R_{r} L_{m}}{L_{r}} i_{s \alpha}-\frac{R_{r}}{L_{r}} \psi_{r \alpha}-p_{p} \omega_{m} \psi_{r \beta} \\
\frac{R_{r} L_{m}}{L_{r}} i_{s \beta}-\frac{R_{r}}{L_{r}} \psi_{r \beta}+p_{p} \omega_{m} \psi_{r \alpha} \\
0 \\
0
\end{array}\right]
\end{aligned}
$$

Denklem (4)'te ifade edilen ileri Euler yaklaşımı kullanılarak GKF gözlemleyicisinde kullanılacak olan ayrık zamanlı motor modeli Denklem (5)'teki gibi elde edilebilir.

$$
\begin{gathered}
\dot{\boldsymbol{x}}_{k} \approx \frac{\boldsymbol{x}_{k+1}-\boldsymbol{x}_{k}}{T} \\
\mathbf{x}_{o, k+1}=\mathbf{I}_{6 \times 6} \times \mathbf{x}_{o, k}+T \times \mathbf{f}_{o}
\end{gathered}
$$

Burada I birim matrisi ifade etmektedir. $T$ ise örnekleme zamanıdır.

$i_{s \alpha}, i_{s \beta}, \psi_{r \alpha}, \psi_{r \beta}, \omega_{m}$ ve $R_{r}$ 'nin eş-zamanlı kestirimi için kullanılan GKF gözlemleyicisine ait genel eşitlikler aşağıda sunulmuştur.

- Doğrusallaştırma adımı

$$
\mathbf{F}_{\mathrm{k}+1 \mid \mathrm{k}}=\left.\frac{\partial \mathbf{f}(\mathbf{x}, \mathbf{u})}{\partial \mathbf{x}}\right|_{\mathbf{x}=\hat{\mathbf{x}}_{\mathrm{k}+1}}
$$

- Kestirim veya zaman güncelleme adımı

$$
\begin{gathered}
\hat{\mathbf{x}}_{\mathrm{k}+1}^{-}=\mathbf{f}\left(\hat{\mathbf{x}}_{\mathrm{k}}, \mathbf{u}_{\mathrm{k}+1}\right) \\
\mathbf{P}_{\mathrm{k}+1}^{-}=\mathbf{F}_{\mathrm{k}+1} \mathbf{P}_{\mathrm{k}} \mathbf{F}_{\mathrm{k}+1}^{\mathrm{T}}+\mathbf{Q}
\end{gathered}
$$

- Ölçüm veya düzeltme güncelleme adımı

$$
\begin{gathered}
\mathbf{K}_{\mathrm{k}+1}=\mathbf{P}_{\mathrm{k}+1}^{-} \mathbf{H}_{\mathrm{k}+1}^{\mathrm{T}}\left[\mathbf{H}_{\mathrm{k}+1} \mathbf{P}_{\mathrm{k}+1}^{-} \mathbf{H}_{\mathrm{k}+1}^{\mathrm{T}}+\mathbf{R}\right]^{-1} \\
\hat{\mathbf{x}}_{\mathrm{k}+1}=\hat{\mathbf{x}}_{\mathrm{k}+1}^{-}+\mathbf{K}_{\mathrm{k}+1}\left(\mathrm{z}_{\mathrm{k}+1}-\mathbf{H}_{\mathrm{k}+1} \hat{\mathbf{x}}_{\mathrm{k}+1}^{-}\right) \\
\mathbf{P}_{\mathrm{k}+1}=\mathbf{P}_{\mathrm{k}+1}^{-}-\mathbf{K}_{\mathrm{k}+1} \mathbf{H}_{\mathrm{k}+1} \mathbf{P}_{\mathrm{k}+1}^{-}
\end{gathered}
$$

Burada $\mathbf{F}_{k+1 \mid k}$ doğrusal olmayan modeli doğrusallaştırmada kullanılan fonksiyonu; $\mathbf{K}_{k+1}$ Kalman kazancin1; $\mathbf{P}_{k+1}^{-}$ve $\mathbf{P}_{\mathrm{k}+1}$ sırasıyla hata kovaryans matrisinin ön ve son değerlerini; $\mathbf{Q}$ sistem $\mathbf{R}$ ise çıkış gürültüsünün kovaryans matrisini ifade etmektedir.

\section{Hız-Algılayıcısız MPTC Tabanlı ASM Sürücüsü}

Önerilen hız-algılayıcısız MPTC temelli sürücü sisteminin blok şeması Şekil 2'de gösterilmiştir. MPTC için gerekli olan akı ve hiz bilgisine ek olarak rotor direnci kestirimi GKF tarafindan gerçekleştirilmiş̦tir. Parametre değişimlerine karşı hızalgılayıcısız ASM sürücüsünün başarımı kestirilen rotor direncinin MPTC sistemine güncellenmesi ile artırılmıştır.

Stator ak1 vektörü, GKF tarafından kestirilen rotor ak1 vektörü ve stator akım vektörü kullanılarak aşağıdaki gibi elde edilir.

$$
\overrightarrow{\hat{\psi}}_{s, k}=\frac{L_{m}}{L_{r}} \overrightarrow{\hat{\psi}}_{r, k}+L_{\sigma} \overrightarrow{\hat{i}}_{s, k}
$$

Şekil 2'de gösterilen "moment ve akı kestirimi" bloğunda $\overrightarrow{\hat{i}}_{s, k}$, $\overrightarrow{\hat{\psi}}_{r, k}$ ve $\overrightarrow{\hat{\psi}}_{s, k}$ kullanılarak, sekiz anahtarlama durumu için $k+1$ anındaki öngörülen stator akıları ve stator akımları aşağıdaki gibi tahmin edilebilir: 


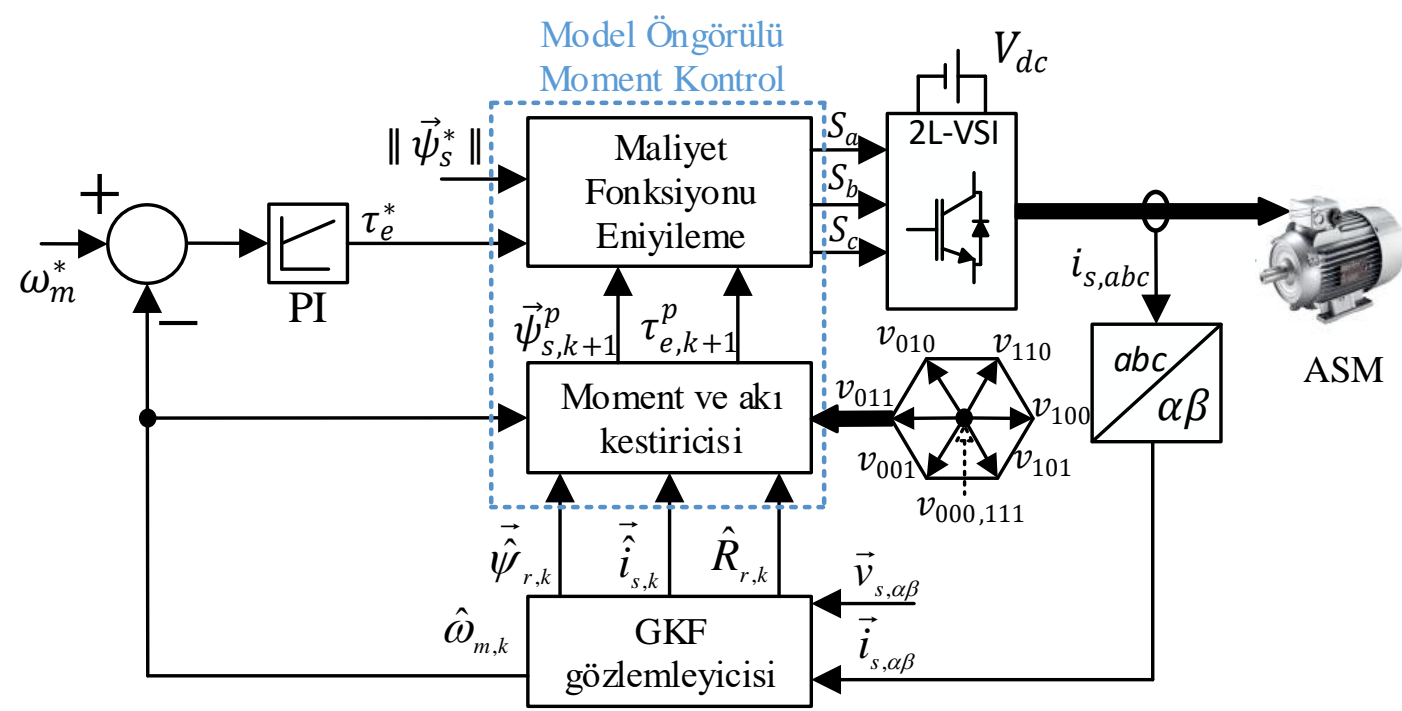

Şekil 2. Hız-algılayıcısız MPTC tabanlı ASM sürücü sistemi (Zerdali vd., 2020)

$$
\begin{gathered}
\vec{\psi}_{s, k+1}^{p}=\overrightarrow{\hat{\psi}}_{s, k}+T \vec{v}_{s, k}^{(j)}+T R_{s} \overrightarrow{\hat{i}}_{s, k} \\
\vec{i}_{s, k+1}^{p}=\left(1-\frac{T}{\hat{T}_{\sigma, k}}\right) \overrightarrow{\hat{i}}_{s, k}+\frac{T}{L_{\sigma}}\left(\left(\frac{k_{r}}{T_{r}}-k_{r} j \omega_{r, k}\right) \overrightarrow{\hat{\psi}}_{r, k}+\vec{v}_{s, k}^{(j)}\right)
\end{gathered}
$$

Burada $j \epsilon\{0,1, \ldots, 7\}, \widehat{T}_{\sigma, k}=L_{\sigma} / \widehat{R}_{\sigma, k}, \hat{R}_{\sigma, k}=R_{s}+k_{r}^{2} \hat{R}_{r, k}$ ve $k_{r}=L_{m} / L_{r}$ 'dir.

Öngörülen $\quad \vec{\psi}_{s, k+1}^{p}$ ve $\vec{i}_{s, k+1}^{p}$ kullanılarak elde edilen elekromanyetik moment aşağıdaki gibi hesaplanır.

$$
\tau_{e, k+1}^{p}=\frac{3}{2} p_{p} \Im m\left\{\left(\vec{i}_{s, k+1}^{p}\right)\left(\vec{\psi}_{s, k+1}^{p}\right)^{*}\right\}
$$

Şekil 2'de verilen "maliyet fonksiyonunun eniyilenmesi" bloğunda en uygun evirici anahtarlama işaretlerinin seçilebilmesi için denklem (16) ile verilen maliyet fonksiyonu kullanılmaktadır.

$$
g=\sum_{h=1}^{N}\left\{\left|\tau_{e}^{*}-\tau_{e, k+h}^{p}\right|+\lambda|| \vec{\psi}_{s}^{*}|-| \vec{\psi}_{s, k+h}^{p}||+I_{m, k+h}\right\}
$$

Burada $\mathrm{N}$ tahmin ufkudur ve $N=1$ olarak seçilmiştir. $\lambda$ akı hatasının ağırlık faktörü, $I_{m}$ aşırı akım koruma terimidir. $I_{m}$ aşağıdaki şekilde tanımlanmıştır.

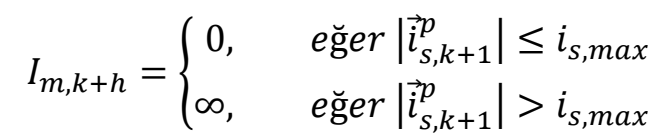

Burada $i_{s, \max }$ stator akımının maksimum genliğidir.

\section{Benzetim Çalışmaları}

$\mathrm{Bu}$ çalışmada önerilen MPTC temelli sürücü MATLAB/Simulink’te tasarlanmış ve doğrulanmıştır. Benzetim çalışmaları Tablo 1'de verilen ASM'ye ait anma değerleri ve parametreler ile gerçekleştirilmiştir. Benzetim çalışmalarında örnekleme zamanı (T) $25 \mu$ s olarak seçilmiştir. Ayrıca, GKF gözlemleyicisi tarafından kestirilen durum ve parametreler sıfır başlangıç değerine sahiptir.
Tablo 1. ASM'nin anma değerleri ve parametreleri

\begin{tabular}{cc||cc}
\hline$P[\mathrm{~kW}]$ & 3 & $p_{p}$ & 2 \\
$f[\mathrm{~Hz}]$ & 50 & $R_{s}[\Omega]$ & 2.283 \\
$V[\mathrm{~V}]$ & 380 & $R_{r}[\Omega]$ & 2.133 \\
$I[\mathrm{~A}]$ & 6.9 & $L_{s}[\mathrm{H}]$ & 0.2311 \\
$n_{m}[\mathrm{dev} / \mathrm{dk}]$ & 1430 & $L_{r}[\mathrm{H}]$ & 0.2311 \\
$\tau_{l}[\mathrm{~N} . \mathrm{m}]$ & 20 & $L_{m}[\mathrm{H}]$ & 0.22 \\
\hline
\end{tabular}

GKF gözlemleyicisinin kestirim başarımını buna bağlı olarak MPTC tabanlı sürücünün kontrol başarımını etkileyen gürültü kovaryans matrisleri ( $\mathbf{Q}$ ve $\mathbf{R})$ ve hata kovaryans matrisinin ( $\mathbf{P}_{0}$ '’n) başlangıç değerleri deneme yanılma yöntemi ile aşağıdaki gibi belirlenmiştir.

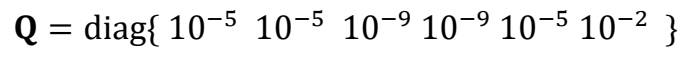

$\mathbf{R}=\operatorname{diag}\left\{\begin{array}{ll}10^{-6} & 10^{-6}\end{array}\right\}$

$\mathbf{P}_{0}=\operatorname{diag}\left\{\begin{array}{llllll}10 & 10 & 10 & 10 & 10 & 10\end{array}\right\}$

Önerilen GKF gözlemleyici tabanlı hız-algılayıcısız MPTC'li ASM sürücü sistemi aşağıdaki senaryolar altında test edilmiş ve doğrulanmıştır.

- Rotor direnci değişimleri altında MPTC temelli sürücünün başarımı.

- Geniş bir hız aralığında farklı yük momentleri altında MPTC temelli sürücünün başarımı.

Benzetim çalışmalarında verilen bütün şekillerde $(\cdot)^{r}$ referans değişimleri, $(\cdot)^{m}$ ölçülen durumları ve $\left({ }^{*}\right)$ kestirilen durumları göstermektedir.

\subsection{Senaryo I}

Bu senaryoda, motor $10 \mathrm{rad} / \mathrm{s}^{\prime}$ lik hız ile $10 \mathrm{~N}$. m'lik yük altında dönerken rotor direnci $t=1 . s$ 'de anma değeri $R_{r n}$ 'den $R_{r n} \times 1.25$ değerine basamak şeklinde artırılmıştır. Daha sonra $t=2$.s'de $R_{r n} \times 1.25$ değerinden anma değeri $R_{r n}$ değerine azaltılmıştır. Bu çalışma şartları için rotor direncinin kestirildiği ve kestirilmediği iki farklı GKF gözlemleyicisi tabanlı MPTC sürücüsünden elde edilen sonuçlar Şekil $3 a$ ve Şekil $3 b$ 'de sunulmuştur. 

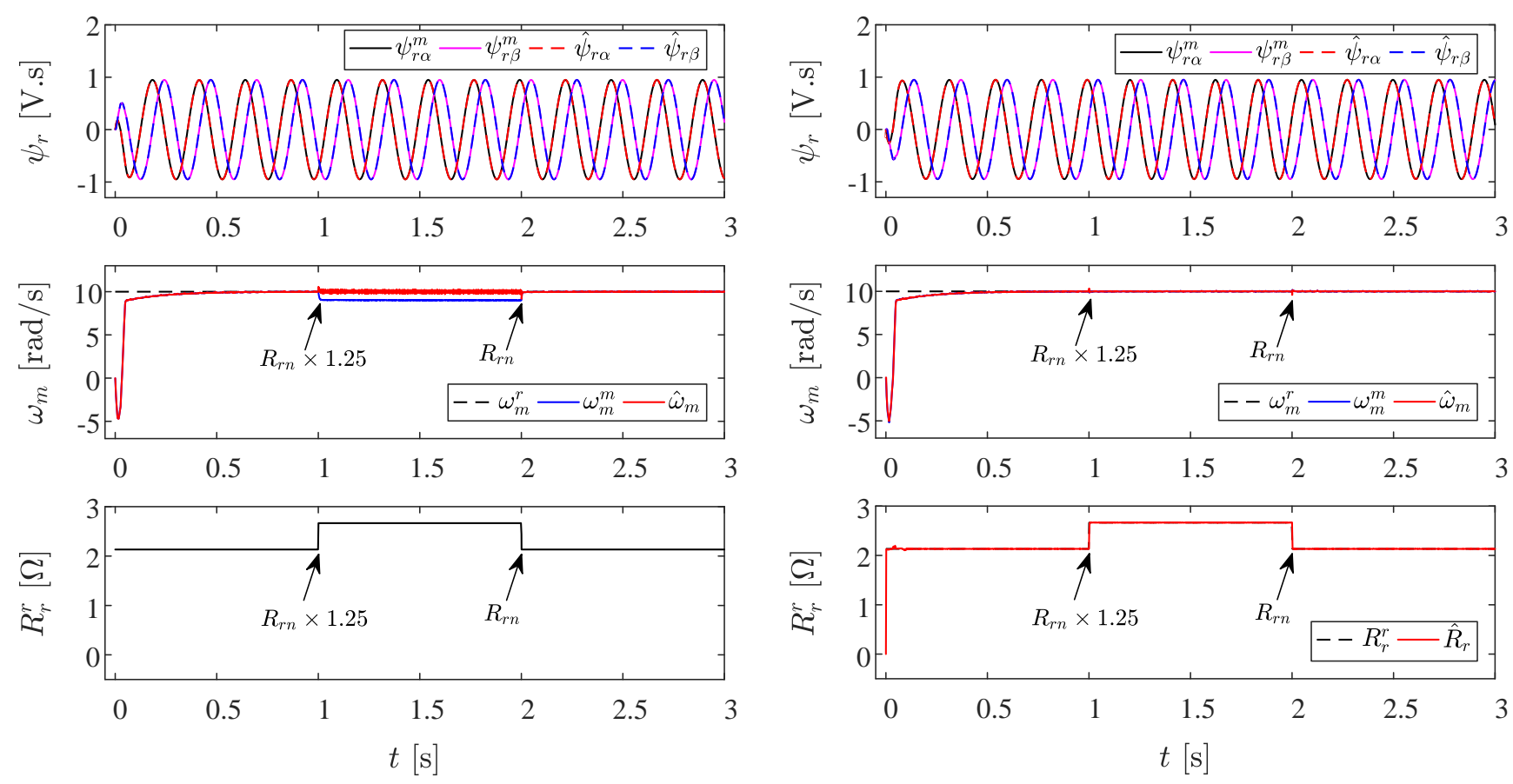

(a)

(b)

Şekil 3. Senaryo I için benzetim sonuçları (a) rotor direnci kestirimi olmadan ve (b) rotor direnci kestirimi ile

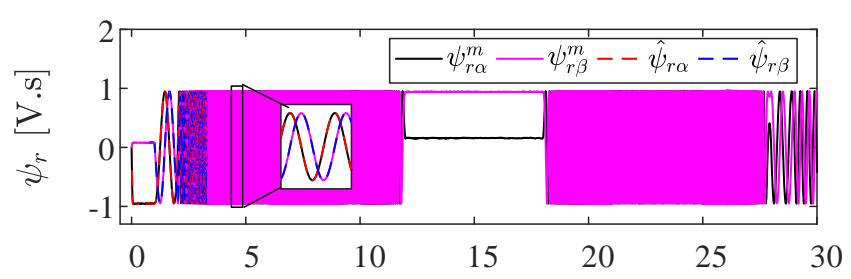
edilmiştir. Elde edilen sonuçlar Şekil 4'te sunulmuştur.

Şekil 3 ve 4'te sunulan sonuçlar önerilen MPTC tabanlı sürücü sisteminin yüksek bir hız kontrol başarımına sahip olduğunu onaylamaktadır.

\section{Sonuç}

$\mathrm{Bu}$ çalışmada rotor direncinin kestirimi ile kontrol başarımı iyileştirilmiş hız-algılayıcısız MPTC tabanlı ASM sürücüsü sunulmuştur. MPTC'nin yüksek başarımlı kontrolü için gerekli olan hız ve akı bilgisine ek olarak rotor direnci GKF tarafindan kestirilmiştir. Kestirilen rotor direnci MPTC sistemi içerisine her bir örnekleme adımında güncellenerek parametre değişimlerinden kaynaklanan bozulmalar azaltılmıştır. GKF algoritması ve bu algoritmayı kullanan MPTC tabanlı ASM sürücüsü yük momenti ve rotor direncinde meydana getirilen değişimler ile oluşturulan zorlayıcı senaryolar altında geniş bir hız aralığında benzetim ortamında test edilmiş ve doğrulanmıştır. Elde edilen benzetim sonuçları, GKF algoritmasının yüksek kestirim başarımına, buna bağlı olarak hız-algılayıcısız MPTC tabanlı ASM sürücüsünün ise yüksek kontrol başarımına sahip olduğunu göstermektedir. Bununla birlikte MPTC sisteminin başarımı ASM'nin stator direnci ve miknatıslama endüktansı gibi diğer elektriksel parametrelerinde de meydana gelebilecek değişimlerden etkilenebilecektir. Bu yüzden daha yüksek kontrol başarımı için bu parametrelerin de kestirilip kontrol sistemine güncellenmesi gerekmektedir.

\section{Kaynakça}

Altınışık, Y. E., Demir, R., \& Barut, M. (2021). Asenkron motorların iyileştirilmiş anahtarlamalı-GKF tabanlı alan yönlendirmeli kontrolü. Nĭgde Ömer Halisdemir Üniversitesi Mühendislik Bilimleri Dergisi, 10(2), 545-552. https://doi.org/10.28948/ngumuh.899447 
Demir, R., \& Barut, M. (2018). Novel hybrid estimator based on model reference adaptive system and extended Kalman filter for speed-sensorless induction motor control. Transactions of the Institute of Measurement and Control, 40(13), 3884-3898. https://doi.org/10.1177/0142331217734631

Lu, Y., \& Zhao, J. (2018). A sliding mode flux observer for predictive torque controlled induction motor drive. 2018 Chinese Control And Decision Conference (CCDC), 3280 3285. https://doi.org/10.1109/CCDC.2018.8407690

Nguyen, N.-D., Nam, N. N. N., Yoon, C., \& Lee, Y. I. (2021). Speed Sensorless Model Predictive Torque Control of Induction Motors using A Modified Adaptive Full-order Observer. IEEE Transactions on Industrial Electronics, 1-1. https://doi.org/10.1109/TIE.2021.3094493

Türker, Ç. G., Karamanakos, P., \& Kennel, R. (2018). Asenkron Makinaların Değişken Anahtarlama Noktalı Model Öngörülü Akım Kontrolü. Fırat Üniversitesi Mühendislik Bilimleri Dergisi, 30(3), 181-187.

Wang, F., Chen, Z., Stolze, P., Stumper, J.-F., Rodríguez, J., \& Kennel, R. (2014). Encoderless Finite-State Predictive Torque Control for Induction Machine With a Compensated MRAS. IEEE Transactions on Industrial Informatics, 10(2), 10971106. https://doi.org/10.1109/TII.2013.2287395

Xie, H., Wang, F., He, Y., Rodriguez, J., \& Kennel, R. (2021). Encoderless Parallel Predictive Torque Control for Induction Machine Using A Robust Model Reference Adaptive System. IEEE Transactions on Energy Conversion, 1-1. https://doi.org/10.1109/TEC.2021.3102305

Yan, L., Dou, M., Hua, Z., Zhang, H., \& Yang, J. (2019). Robustness Improvement of FCS-MPTC for Induction Machine Drives Using Disturbance Feedforward Compensation Technique. IEEE Transactions on Power Electronics, 34(3), 2874-2886. https://doi.org/10.1109/TPEL.2018.2842743

Yan, L., \& Song, X. (2020). Design and Implementation of Luenberger Model-Based Predictive Torque Control of Induction Machine for Robustness Improvement. IEEE Transactions on Power Electronics, 35(3), 2257-2262. https://doi.org/10.1109/TPEL.2019.2939283

Yin, S., Huang, Y., Xue, Y., Meng, D., Wang, C., Lv, Y., Diao, L., \& Jatskevich, J. (2019). Improved Full-Order Adaptive Observer for Sensorless Induction Motor Control in Railway Traction Systems Under Low-Switching Frequency. IEEE Journal of Emerging and Selected Topics in Power Electronics, $1-1$. https://doi.org/10.1109/JESTPE.2019.2898875

Zerdali, E., \& Demir, R. (2021). Speed-sensorless predictive torque controlled induction motor drive with feed-forward control of load torque for electric vehicle applications. Turkish Journal of Electrical Engineering \& Computer Sciences, 29(1), 223-240.

Zerdali, E., Demir, R., \& Barut, M. (2020). Speed-Sensorless FCS-PTC Based Induction Motor Drive Capable of Disturbance Rejection. 2020 2nd Global Power, Energy and Communication Conference (GPECOM), 170-175. https://doi.org/10.1109/GPECOM49333.2020.9247917

Zhao, Z., Ruan, Z., Meng, D., Xue, Y., \& Gu, C. (2019). Sliding Mode Observer Based Sensorless Model Predictive Current e-ISSN: 2148-2683
Control for Induction Motor. 2019 IEEE 2nd International Conference on Power and Energy Applications (ICPEA), 8488. https://doi.org/10.1109/ICPEA.2019.8818497 